\title{
A Distributed Storage System for a Video-on-Demand Server
}

\author{
Alice Bonhomme and Loïc Prylli \\ LHPC/ ENS Lyon, 69364 Lyon, France \\ \{Alice.Bonhomme, Loic.Prylli\}@ens-lyon.fr
}

\begin{abstract}
The aim of this paper is to present the design of a distributed storage system for a video server. The main goal is to support good faulttolerance capabilities (no single point of failure, and no perturbation of the clients at the time the failure occurs) while supporting a high-number of video streams.
\end{abstract}

\section{Introduction}

The aim of this paper is to present the design of a distributed storage system for a video server. A distributed architecture is quite natural for a video server, given the intrinsic parallelism provided by independent clients on one hand, and the possibility of easily fetching multiple blocks in parallel for a given stream on the other hand. We use a "PC type" cluster, which provides a good performance/price ratio. An important aspect of video servers is the continuity of service even in case of a hardware failure, a distributed architecture provides the required redundancy to handle such failure.

Our goal was to minimize the resource overhead, so a parity strategy is used to manage redundancy rather than mirroring blocks. Then to handle failures without any visible perturbation in the service, the parity block is systematically read just in case (the cost of doing this is low enough). For the intended target of this video-on-demand server, no cache strategy seems possible, so all data to the clients is systematically fetched from disk.

\section{Related Works}

The subject of video servers (see [GVK ${ }^{+}$95, GM98, TMDV96]) has been explored in both theory and practice. This is still a active subject because of the diversity of goals. We list below different distributed implementations:

RIO: at UCLA MSB97 is based on random allocation of striping nodes. This makes it possible to support a wide range of multimedia needs.

MITRA: at USC [GZS ${ }^{+}$] ] focuses on optimizing the throughput by precisely controlling the data placement to optimize the movements of the disk heads.

Server Array: at Eurecom BB96a is able to cope with many types of heterogeneity : number of nodes or disks, striping strategy, reliability schemes. 
Tiger: at Microsoft $\left[\mathrm{BB}^{+} 96 \mathrm{~b}\right]$ implements a distributed stream scheduling, replicates data blocks and distributes each copy among a subset of nodes.

From a fault tolerance point of view, these video server implementations exhibits various strategies (parity/mirroring, cf. [GLP98]) and different degrees of fault tolerance (disk, node, network). Tiger uses a distributed replication of the blocks. Mitra chooses to replicate the whole disk and to systematically read on both disks. The Server Array is designed to support any kind of failures using a combination of both strategies. In RIO, the replication scheme of the blocks seems to be more adapted. However, excepted Tiger, all the other prototypes suffer from a single point of failure due to the presence of a meta server, which is in charge of the client connection and the stream scheduling.

\section{Overview of the Complete Video Server}

The video server (cf. Figure 11) is a cluster of PCs with local storage units. These PCs are interconnected using a Myrinet network. This internal network is independent from the distribution network.

The video server is divided into three parts. The first module manages RTSP client sessions via the distribution network. The second module schedules all real time IO operations and gathers data from the third module: the cluster file system (CFS). This CFS module relies on the internal network (using the GM1 communication library) to manage the distributed storage (open, close, read and write operations to perform on the cluster nodes). It mainly consists of one IOM

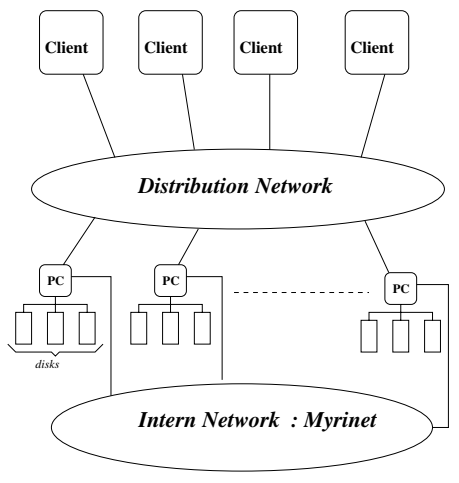

Fig. 1. The video server hardware architecture (IO Manager) on each node. In fact, all the software components are distributed among all the server nodes.

\section{The Cluster File System}

Video data placement and management. We use a striping strategy. A video file is divided into blocks of equal size, distributed among the nodes of the cluster or among a subset of the cluster nodes. Furthermore, for fault tolerance issues, we have parity blocks stored on a node of the subset. When a file is stripped among $n$ nodes, this requires $1 / n$ additional space to store the parity blocks. The distribution information for each file is stored in a global table. This table is persistent, and is replicated on each node. To keep it consistent across the nodes, it is accessed through a mutual exclusion scheme. This table also permits

${ }^{1}$ GM: message passing system from Myricom, Inc (http://www.myri.com/GM) 
to deal with permission problems using a counter for read sessions and write sessions. A local persistent table on each node gives the information about the location of data stored on the local disks.

Implementation of distributed IO. The read function posts an asynchronous read on the distributed file. This function returns an operation descriptor that allows the user to check for the completion of the operation. A read operation is posted from a client to its local IOM, which distributes it among the involved nodes. If necessary, the IOM posts a local read using the local IO subsystem. Remote requests are handled through the internal network. Besides the file's data blocks, the parity block is also systematically requested. If a failure occurs, the parity is already available and no additional delay is needed to get the parity information from the parity node. A status function allows to check for completion of the operation. If all the data has been retrieved, the function returns with a completed status. If one response is missing, the missing data is reconstructed using the parity information and the function returns with the completed status. If more than one response is missing, the function returns with a "not ready" status. The write operation is essentially similar to the read operation. The difference is mainly the addition of the parity blocks generation before sending the request to the remote nodes.

The IOM scheme. The IOM manages at the same time the client requests, the remote IOMs requests and the local accesses. The client requests and the local accesses are stored into two internal queues, the remote IOM requests are stored into a queue fed to the internal network. The IOM scheme consists of polling on each queue and handling the requests. Thus, depending on the request, the IOM sends messages to remote IOMs, calls the local IO subsystem to perform local accesses or updates some local information. The IOM must also keep the context of each client and each operation. Finally it is responsible of the fault tolerance management (cf. Section 5)

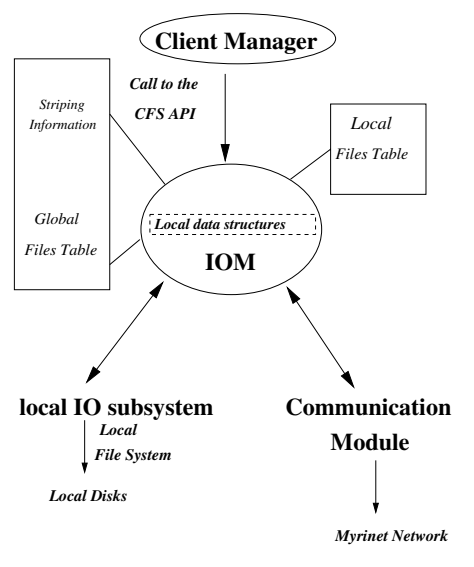

Fig. 2. Interactions between the modules of the CFS

\section{Fault Tolerance Management}

Within the distributed storage system, it is important that the failure of some component (node, disk, network link) does not cause a server crash. Therefore, we designed a strategy to support any failure (no single point of failure). 
Distinction between transient problem and permanent failure. In case of a transient problem lasting less than $n$ seconds (node disconnected from the network for example), the corresponding node is considered as potentially failed, the missing data are computed thanks to parity blocks. Then if the problem disappears, the node comes back in the server without any special treatment. In case a fault is lasting more than $n$ seconds, we rely on the unreachable notification mechanisms of GM to avoid false detection, and the other nodes can consider the unreachable node as permanently failed (each node regularly sends probe messages in order to detect failures even if there are no other useful communications required between the nodes).

Mutual exclusion algorithm. We use a classical algorithm based on the logical clocks defined by Lamport. In order to support a node failure, we modify this algorithm by checking the state of a node that is not responding to mutual exclusion requests or that has failed while owning an exclusive access.

Node recovery. When a node fails, it is important to repair this node without having to stop the server. The recovering node proceeds in several steps: it gets the current global files table and striping table after gaining exclusive access to them, then it can use the striping table and can compare it to its local table to detect video data that needs to be reconstructed, finally it sends read requests to the other nodes to reconstruct the missing data using the parity blocks.

\section{Experimental Results}

We validated the storage subsystem on a 3-nodes cluster made up of Intel biprocessors connected with a Myrinet network. Each node has four UltraWide/7200 RPM disks in a RAID 0 configuration, accessed using the NTFS file system. What we want to compute is the maximum number of streams for

\begin{tabular}{|l|c|c|}
\hline & without fault & with fault \\
\hline 1 client node & 34 & 55 \\
\hline 2 client nodes & $2 \times 32$ & $2 \times 35$ \\
\hline 3 client nodes & $3 \times 23$ & - \\
\hline
\end{tabular}

Fig. 3. Number of $4 \mathrm{Mbit} / \mathrm{s}$ streams supported on a 3-nodes cluster.

a given bit-rate. We use a test program

that uses the CFS to retrieve streams and checks that the real time constraints are met for each block. This allows to experimentally compute the maximum number of streams supported. This experiment is done by executing client requests from one or several nodes, and in presence of a node fault or not (in case of one failure, there can be at most 2 client nodes). The results are shown in Table 3.

The maximum number of streams globally supported by the server is around 70 which can be shown to match the above hardware using the computation given in BP00]. The lower part of the array is close to the maximum capacity of the server limited by the local IO capacity. The performance with a fault is better than without, because the redundancy of getting one extra remote block is avoided. 


\section{Conclusion}

The aim of this paper was to describe the overall design of a distributed storage system targeted at video. The choices that have been made are consistent with the intended target: reliably delivering video while supporting as many streams as possible even in case of failure.

If redundancy strategies and fault recovery have been well studied in previous works, in practice the problem of fault detection of a host must be correctly handled: the design should guarantee a consensus between all other hosts before deciding that another one has failed (for the purpose of mutual exclusion), but this consensus can take time. The solution proposed here allows the delivery of ongoing streams without perturbation (handling parity blocks does not depend on mutual exclusion and thus on host fault detection), independently of the time needed to detect reliably an host failure.

\section{References}

[BB96a] C. Bernhardt and E. Biersack. High-Speed Networking for Multimedia Applications, chapter The Server Array: A Scalable Video Server Architecture. Kluwer, 1996.

$\left[\mathrm{BB}^{+}\right.$96b] William J. Bolosky, Joseph S. Barrera, et al. The Tiger video fileserver. In Proceedings of the Sixth International Workshop on Network and Operating System Support for Digital Audio and Video. IEEE Computer Society, April 1996.

[BP00] Alice Bonhomme and Loic Prylli. A distributed storage system for a videoon-demand server. Technical Report 2000-16, LIP, ENS Lyon, France, April 2000.

[GLP98] L. Golubchik, J. Lui, and M. Papadopouli. A survey of approaches to fault tolerant design of VOD servers: Techniques, analysis and comparison. Parallel Computing, 24(1):123-155, 1998.

[GM98] S. Ghandeharizadeh and R. Muntz. Design and implementation of scalable continuous media servers. Parallel Computing, 24:91-122, 1998.

[GVK $\left.{ }^{+} 95\right]$ D.J. Gemmel, H.M. Vin, D.D. Kandlur, P.V. Rangan, and L. Rowe. Multimedia storage servers: A tutorial and survey. IEEE Computer, 28(5):4049, November 1995.

[GZS $\left.{ }^{+} 97\right]$ S. Ghandeharizadeh, R. Zimmermann, W. Shi, R. Rejaie, D. Ierardi, and A.W Li. Mitra : A scalable continuous media server. Multimedia Tools and Applications Journal, 5(1):79-108, July 1997.

[MSB97] R. Muntz, J.R Santos, and S. Berson. RIO: A real-time multimedia object server. ACM Performance Evaluation Review, 25(2):29-35, September 1997.

[TMDV96] R. Tewari, R. Mukherjee, D.M. Dias, and H.M. Vin. Design and performance tradeoffs in clustered video servers. In the IEEE international Conference on Multimedia Computing and Systems (ICMCS'96), pages 144-150, May 1996. 